Urol. Prax. 2021 · 23:151-155

https://doi.org/10.1007/s41973-021-00152-z

Accepté: 21. Septembre 2021

Publié en ligne: 20. Octobre 2021

(c) The Author(s) 2021

\section{Introduction}

La gynécomastie est définie comme une augmentation du tissu glandulaire mammaire chez l'homme.

Sa prévalence est estimée entre 32 et $65 \%$ et varie largement avec l'âge et la définition utilisée. Trois pics d'incidence sont décrits: la gynécomastie se développe chez 65 à $90 \%$ des nouveau-nés, 22 à $69 \%$ des adolescents et 36 à $57 \%$ des hommes entre la cinquième et septième décennie de vie [1].

La présentation clinique typique est une augmentation du tissu glandulaire péri-aréolaire. Elle peut être d'apparition rapide ou progressive, bilatérale ou unilatérale, indolore ou douloureuse [2]. Une anamnèse familiale oncologique est essentielle, étant donné que la mutation du BRCA2 est le facteur de risque principal pour un cancer du sein chez l'homme [3].

La gynécomastie est un problème fréquent, qui affecte la qualité de vie des patients au vu des aspects psychosociaux et possibles douleurs associées [4]. De plus, elle peut signaler une pathologie sous-jacente que le praticien doit identifier afin d'instaurer le traitement adéquat.

Le but de l'article est de résumer la pathophysiologie, les étiologies principales, lévaluation clinique et les examens complémentaires ainsi que les bases de la prise en charge.

\section{Pathophysiologie}

Histologiquement, la gynécomastie se caractérise par une hyperplasie des canaux galactophores et du stroma fibro-musculaire.

Julien Blanc - Beat Roth · Arnas Rakauskas

Departement of Urology, University of Lausanne, Lausanne, Suisse

\title{
Gynécomastie en pratique clinique pour les urologues
}

Le tissu glandulaire contient des récepteurs aux œstrogènes aussibien qu'aux androgènes; l'activation des récepteurs aux œstrogènes mène à la prolifération du tissuglandulaire tandis quel'activation des récepteurs aux androgènes inhibe la croissance et la différenciation glandulaire. Le mécanisme exact amenant à une gynécomastie n'est encore que partiellement compris. Cependant, un dérèglement entre la concentration des œstrogènes et des androgènes semble en être la cause principale $[1,5]$.

Chez l'homme, 15\% de l'œstradiol et la majorité de la testostérone, l'hormone masculine la plus importante, sont produites par les testicules [6]. Dans le sang, la testostérone est majoritairement liée à la globuline liant les hormones sexuelles (SHBG), produite par le foie. La partie libre étant la partie bioactive, passe du sang aux organes cibles. La plupart de la testostérone est convertie par l'aromatase en œstrogènes dans les organes cibles: gonades, tissu adipeux et tissu mammaire.

De nombreuses pathologies ou médicaments peuvent influencer une des différentes étapes amenant à une altération de l'équilibre entre œstrogènes-testostérone résultant en gynécomastie (•Tab. 1).

\section{Étiologie}

\section{Causes hormonales}

En principe, trois types de déséquilibre hormonal peuvent se manifester: la diminution de la concentration de la testostérone, l'augmentation de la concentration des œstrogènes ou l'augmentation de la concentration de la testostérone et des œstrogènes conjointement. Le - Tab. 2 résume les causes principales.

\section{Diminution de la testostérone}

Lorigine de la diminution du taux de testostérone peut être primaire ou secondaire. Dans le premier cas, les testicules sont hypoactifs et la diminution de la testostérone circulante causera une augmentation de la production de l'hormone lutéinisante $(\mathrm{LH})$ qui à son tour stimulera la fonction de l'aromatase. Les pathologies ayant une atteinte directe sur la fonction testiculaire comme un trauma testiculaire, une orchite bilatérale, les tumeurs testiculaires, la radiothérapie testiculaire ou chimiothérapie sont les causes principales. Dans les causes plus rares d'hypogonadisme primaire, nous retrouvons le syndrome de Klinefelter, des défauts enzymatiques affectant la production de la testostérone ou des délétions chromosomiques.

Dans les causes secondaires, le déficit de production de la testostérone est dû à un défaut de l'axe hypothalamohypophysaire avec diminution de la production de l'hormone de libération de gonadotrophines hypophysaires $(\mathrm{GnRH})$ ou de LH. En conséquence, la testostérone sanguine est diminuée et la LH normal, voire basse. Lélévation du taux de prolactine n'est pas associée directement à une gynécomastie, mais elle peut induire une suppression de la production de la GnRH amenant à un hypogonadisme secondaire. Finalement, une insuffisance rénale chronique peut aussi amener à une déficience en testostérone causant une dysfonction au niveau gonadique ou hypothalamo-hypophysaire.

\section{Augmentation des œstrogènes}

Plusieurs causes peuvent augmenter la production des œstrogènes. Une origine fréquente est une augmentation des œstrogènes à la suite de l'aromatisation 


\begin{tabular}{|c|c|}
\hline Cause & Exemples \\
\hline Physiologique & Nouveau-né, adolescence, vieillissement \\
\hline Hypogonadisme & Primaire, secondaire \\
\hline Médicamenteuse & Anti-androgène, kétoconazole, etc. \\
\hline \multirow[t]{2}{*}{ Néoplasme } & Testiculaire (germinale, non-germinale) \\
\hline & Extra-testiculaire \\
\hline Pathologie thyroïdienne & Hypo-, hyperthyroïdie \\
\hline Pathologie hépatique et rénale & Cirrhose, insuffisance rénale chronique \\
\hline Génétique & $\begin{array}{l}\text { Syndrome de Klinefelter, syndrome de Kennedy, hyperplasie } \\
\text { congénitale des surrénales, etc. }\end{array}$ \\
\hline Environnementale/exposition & Anabolisant, cannabis, alcool, exposition à des œstrogènes \\
\hline Autre & Obésité, jeûne, idiopathique \\
\hline
\end{tabular}

périphérique de la testostérone en œstrogènes par l'aromatase. Celle-ci se retrouve principalement dans le tissu adipeux. Par conséquent, l'obésité peut amener à une augmentation de la concentration en œstrogènes.

Chez le patient présentant une cirrhose, nous retrouvons une augmentation de l'aromatisation hépatique mais aussi une augmentation de la production de la SHBG amenant à une diminution de la testostérone libre. Lalcoolisme chronique, la consommation de cannabis et la prise exogène des œstrogènes sont aussi associés à une augmentation des œstrogènes qui peut se manifester par une gynécomastie.

\section{Origines mixtes}

Il existe d'autres pathologies qui peuvent augmenter le taux de la testostérone et des œstrogènes conduisant à une gynécomastie. Il s'agit des tumeurs testiculaires à cellules de Leydig ou Sertoli, les tumeurs germinales, l'hyper ou hypothyroïdisme, la prise de stéroïde androgénique anabolisant et des syndromes congénitaux.

Les tumeurs à cellules de Leydig sont les tumeurs stromales les plus fréquentes, mais une cause rare de gynécomastie. La tumeur étant biologiquement active, présente une sécrétion accrue de testostérone et d'œstradiol. La majorité de ces tumeurs, $90 \%$, sont bénignes et uniquement $10 \%$ sont malignes. Ces tumeurs sont plus fréquentes chez les enfants (entre 5 et 10 ans) ou chez l'homme d'âge moyen (entre 30 et 60 ans) [1,5-7]. Les tumeurs à cellules de Sertoli sont rares et uniquement un tiers des patients présente une gynécomastie. Le mécanisme sous-jacent n'est pas encore élucidé. L'hypothèse est une production directe d'œstrogènes par les cellules de Sertoli [7].

Les tumeurs testiculaires germinales, surtout celles contenant une composante de choriocarcinome, peuvent provoquer une gynécomastie, En effet, ces tumeurs sécrètent de l'hormone chorionique gonadotrope humain (hCG) qui, en raison de son homologie structurelle à la $\mathrm{LH}$, à son tour stimule la production de testostérone et l'activité de l'aromatase amenant à un excès d'œstrogènes. Étant donné que ce type de tumeurs est souvent malin et agressif, la survenue de la gynécomastie est plus rapide [1, 5].

En ce qui concerne les pathologies thyroïdiennes, aussibien l'hyperthyroïdisme que l'hypothyroïdisme peuvent provoquer une gynécomastie. L'augmentation des hormones thyroïdiennes amène à une augmentation de la SHBG. La diminution de la testostérone libre amènera à son tour à la stimulation de la production de LH qui favorise l'aromatisation périphérique d'androgènes en œstrogènes. Dans le cas de l'hypothyroïdisme, la diminution de testostérone est probablement due à l'augmentation de la production de la prolactine par l'hormone thyréotrope (TRH).

De plus, la prise de stéroïdes androgéniques anabolisants peut amener à une gynécomastie principalement en raison de l'aromatisation périphérique de ces derniers en œstrogènes.

Dans les causes plus rares de gynécomastie dues à l'augmentation de la testostérone et des œstrogènes circulants,

\begin{tabular}{|c|c|}
\hline \multirow{4}{*}{$\begin{array}{l}\text { Diminution de } \\
\text { la testostérone }\end{array}$} & Hypogonadisme primaire \\
\hline & $\begin{array}{l}\text { Hypogonadisme secon- } \\
\text { daire }\end{array}$ \\
\hline & Prolactinémie \\
\hline & $\begin{array}{l}\text { Insuffisance rénale chro- } \\
\text { nique }\end{array}$ \\
\hline \multirow{5}{*}{$\begin{array}{l}\text { Augmentation } \\
\text { des œstrogènes }\end{array}$} & Obésité \\
\hline & Alcoolisme chronique \\
\hline & Cirrhose \\
\hline & Fstrogènes exogènes \\
\hline & Cannabis \\
\hline \multirow[t]{6}{*}{ Mixtes } & $\begin{array}{l}\text { Tumeur à cellules de } \\
\text { Leydig }\end{array}$ \\
\hline & $\begin{array}{l}\text { Tumeur à cellules de } \\
\text { Sertoli }\end{array}$ \\
\hline & Tumeur germinales \\
\hline & Hyper-/hypothyroïdisme \\
\hline & Androgène exogène \\
\hline & Génétique \\
\hline
\end{tabular}

nous retrouvons le syndrome de Kennedy (augmentation des triplets CAG dans le gène du récepteur aux androgènes amenant à une sensibilité diminuée aux androgènes) associé à des troubles neuromusculaires et le syndrome d'insensibilité aux androgènes (défaut génétique du récepteur aux androgènes) [1].

\section{Causes médicamenteuses}

Plusieurs médicaments ont été impliqués dansle développement d'une gynécomastie [8].

Les médicaments qui provoquent une gynécomastie d'une manière fréquente sont les traitements de suppression des androgènes utilisés dans le cadre des carcinomes prostatiques. Les anti-androgènes (flutamide, bicalutamide, nilutamide) sont les traitements avec la plus haute incidence de gynécomastie (entre 40 et $70 \%$ ), suivis par les analogues de la $\mathrm{GnRH}$ (goséréline, leuproréline). En général, la gynécomastie apparaît dans la première année de traitement [9-12].

Les inhibiteurs de la $5 a$-réductase (finastéride, dutastéride), qui sont utilisés pour les troubles de la vidange vésicale dans le cadre d'une hypertrophie prostatique, peuvent être associés à la gyné- 
comastie même si moins fréquemment ( $0,4 \%$ pour le finastéride) [9].

Finalement, plusieurs autres traitements sont associés au développement d'une gynécomastie. Cependant, le niveau d'évidence est souvent faible, hormis pour la spironolactone, le kétoconazole, la cimétidine, l'hormone de croissance et les gonadotrophines.

\section{Diagnostic}

Lanamnèse est essentielle pour le diagnostic de la gynécomastie. La durée de l'apparition et la rapidité de l'instauration, les symptômes associés comme la douleur, des saignements ou des écoulements sont à rechercher. Lanamnèse médicamenteuse devrait être précise y compris la prise de substance illicite ou d'anabolisant. Enfin, une anamnèse oncologique recherchera une histoire familiale de cancer du sein, de la prostate ou testiculaire.

À l'examen clinique, la gynécomastie devrait être différenciée de la lipomastie. L'examen clinique est réalisé au début en position assise puis en décubitus dorsal. La prolifération du tissu glandulaire se traduit à la palpation sous-aréolaire par un tissu plus ferme que celui de la prolifération du tissu adipeux. Lors de l'examen clinique, des signes évocateurs de cancer mammaire comme une rétraction cutanée, une " peau d'orange ", une asymétrie du tissu glandulaire, un écoulement/saignement du mamelon seront recherchés systématiquement. Pour terminer, l'examen clinique est complété par une inspection des organes génitaux à la recherche d'une masse testiculaire évoquant un cancer testiculaire.

En pratique, la réalisation d'une échographie testiculaire et la mesure des marqueurs tumoraux s'imposent, car toutes les tumeurs ne sont pas palpables. L'Association Européenne d'Andrologie propose de réaliser un bilan hormonal de base avec mesure de la testostérone, $17 \beta$ estradiol, SHBG, LH, FSH, TSH, prolactine, hCG, AFP [1]. Si l'anamnèse ou l'examen clinique sont évocateurs d'un cancer mammaire, une mutation du gène BRCA sera à rechercher. Celle-ci est le facteur de risque principal dans le dé-

Urol. Prax. 2021·23:151-155 https://doi.org/10.1007/s41973-021-00152-z

(c) The Author(s) 2021

\section{J. Blanc $\cdot$ B. Roth $\cdot$ A. Rakauskas \\ Gynécomastie en pratique clinique pour les urologues}

\section{Résumé}

La gynécomastie est fréquemment retrouvée dans la pratique clinique de l'urologue ou du médecin généraliste. D'un point de vue physiopathologique, elle résulte d'un déséquilibre entre la concentration des œstrogènes et des androgènes. De multiples pathologies et traitements peuvent amener à cette perte d'équilibre. Il est important pour le clinicien de reconnaitre les signes de danger qui doivent faire suspecter une néoplasie maligne primaire du tissus mammaire ou secondaire ayant amené à la gynécomastie. Un bilan biologique de base permet le plus souvent de trouver la cause de la gynécomastie. Le traitement est le plus souvent sa cause. Pour certain cas particulier un traitement par le tamoxifène ou la radiothérapie peut être proposé. La chirurgie intervient lors de l'échec du traitement conservateur.

\section{Mots clés}

Urologie - Gynécomastie · Pratique clinique . Troubles hormonaux · Prise en charge

\section{Gynäkomastie in der klinischen Praxis für Urologen}

\section{Zusammenfassung}

Der Urologe und der Allgemeinmediziner begegnen häufig der Gynäkomastie in der klinischen Praxis. Aus pathophysiologischer Sicht entsteht sie aufgrund einer Störung des Östrogen-/Androgengleichgewichts. Verschiedene Krankheitsbilder und Therapien können zu diesem Ungleichgewicht führen. Es ist für den Kliniker wichtig, die Gefahrensignale zu erkennen, die auf einen primären oder sekundären malignen Tumor des Brustdrüsengewebes als Ursache der Gynäkomastie hinweisen. Die Ursache der
Gynäkomastie kann oft durch grundlegende Laboranalysen ermittelt werden. Am häufigs ten ist eine Therapie die Ursache. In manchen Einzelfällen kann eine Tamoxifenbehandlung oder eine Radiotherapie angeboten werden. Die chirurgische Behandlung kommt bei Versagen der konservativen Therapie zum Einsatz.

\section{Schlüsselwörter}

Urologie · Gynäkomastie · Klinische Praxis . Hormonelle Störungen · Betreuung veloppement de cancer mammaire chez l'homme [3].

De plus, des modalités d'imagerie complémentaires peuvent être indiquées à la recherche de tumeurs extra-testiculaires.

\section{Traitement}

Le traitement de la gynécomastie concerne principalement celui de la cause sousjacente.

Les stratégies thérapeutiques comprennent l'utilisation du tamoxifène (un modulateur sélectif des récepteurs aux œstrogènes), l'anastrozole (un inhibiteur de l'aromatase), la radiothérapie et la chirurgie [5].

Chez les patients présentant une gynécomastie induite par le traitement antiandrogène, le traitement par le tamoxifène $20 \mathrm{mg}$ journalier a été évalué com- me le plus efficace dans la prévention de l'apparition ou du traitement de la gynécomastie [5, 13, 14]. D’autre part, ce type de traitement augmente le risque des évènements cardiovasculaires ischémiques et thromboemboliques.

Chez le patient présentant une contreindication au traitement par le tamoxifène, la radiothérapie est l'option de choix, l'avantage étant la courte durée du traitement. Par contre, les patients devraient être informés d'une possible cardiotoxicité [15-17]. En revanche, elle devrait être évitée chez le patient jeune au vu du risque de développement d'un cancer mammaire secondaire [18].

Les mêmes stratégies thérapeutiques sont utilisées dans le cadre des gynécomasties idiopathiques ou quand la cause primaire ne peut pas être corrigée. Un traitement par le tamoxifène 10-20 mg journalier pendant 3-9 mois 
amène en général à une disparition de la gynécomastie dans $90 \%$ des cas [19]. L'anastrozole est en général moins efficace [20] sauf cas particuliers (chez les patients présentant des syndromes familiaux avec excès de l'aromatase [21]). La substitution de testostérone peut être proposée si la cause est un hypogonadisme primaire avec une diminution prouvée de la testostérone [1].

C'est à souligner que les traitements médicamenteux sont plus efficaces dans les deux premières années depuis l'apparition des symptômes. En raison de la fibrose des tissus, l'efficacité du traitement diminue avec le temps [5, 22].

La chirurgie peut être proposée aux patients ne répondant pas aux traitements médicamenteux ou à la radiothérapie ou souhaitant une correction rapide de la gynécomastie [1]. En fonction de la taille et de la localisation du tissu glandulaire, différentes techniques chirurgicales sont disponibles. Pour les gynécomasties légères la liposuccion est le premier traitement proposé. Pour des gynécomasties modéré une mastectomie sous-cutanée par un abord peri-aréolaire minimale avec par la suite application d'un bandage compressif est efficace et permet un bon résultat cosmétique. Cette approche peut être combiné à une liposuccion. Lors des gynécomasties plus importantes une approche chirurgicale avec excision du surplus de peau plus ou moins reconstruction par lambeau est utilisée [23-25].

\section{Conclusion}

La gynécomastie masculine est fréquente dans la population. En premier lieu, une tumeur mammaire ou testiculaire devrait être exclue. Un bilan hormonal de base permet de trouver une cause sous-jacente dans la majorité des cas. Pour la gynécomastie induite par les traitements de suppression androgénique ou chez la gynécomastie idiopathique, le traitement de choix est le tamoxifène avec la radiothérapie comme alternative. La gynécomastie invalidante et réfractaire à ces traitements est traitée chirurgicalement.

\section{Adresse de correspondance}

\section{Julien Blanc}

Departement of Urology, University of

Lausanne

Rue du Bugnon 46, CHUV, 1011 Lausanne, Suisse

Julien.Blanc@chuv.ch

Funding. Open access funding provided by University of Lausanne

\section{Conformité aux directives éthiques}

Conflit d'intérêts J. Blanc, B. Roth et A. Rakauskas déclarent ne pas avoir de conflit d'intérêt.

Pour cet article, les auteurs n'ont réalisé aucune étude sur les humains ou les animaux. Les études réalisées étaient conformes aux normes éthiques indiquées dans chaque cas.

Open Access. This article is licensed under a Creative Commons Attribution 4.0 International License, which permits use, sharing, adaptation, distribution and reproduction in any medium or format, as long as you give appropriate credit to the original author(s) and the source, provide a link to the Creative Commons licence, and indicate if changes were made. The images or other third party material in this article are included in the article's Creative Commons licence, unless indicated otherwise in a credit line to the material. If material is not included in the article's Creative Commons licence and your intended use is not permitted by statutory regulation or exceeds the permitted use you will need to obtain permission directly from the copyright holder. To view a copy of this licence, visit http://creativecommons.org/licenses/by/4.0/.

\section{Références}

1. Kanakis GA, Nordkap L, Bang AK, Calogero AE, Bártfai G, Corona G et al (2019) EAA clinical practice guidelines-gynecomastia evaluation and management. Andrology 7:778-793. https://doi. org/10.1111/andr.12636

2. Costanzo PR, Pacenza NA, Aszpis SM, Suárez SM, Pragier UM, Usher JGS et al (2018) Clinical and etiological aspects of gynecomastia in adult males: a multicenter study. Biomed Res Int 2018:8364824. https://doi.org/10.1155/2018/8364824

3. Abdelwahab Yousef AJ (2017) Male breast cancer: epidemiology and risk factors. Semin Oncol 44:267-272. https://doi.org/10.1053/j. seminoncol.2017.11.002

4. Fagerlund $A$, Lewin R, Rufolo $G$, Elander $A$, Santanelli di Pompeo F, Selvaggi G (2015) Gynecomastia: a systematic review. J Plast Surg Hand Surg 49:311-318. https://doi.org/10.3109/ 2000656X.2015.1053398

5. Narula HS, Carlson HE (2014) Gynaecomastia-pathophysiology, diagnosis and treatment Nat Rev Endocrinol 10:684-698. https://doi.org/ 10.1038/nrendo.2014.139

6. Baumgarten L, Dabaja AA (2018) Diagnosis and management of gynecomastia for urologists. Curr
Urol Rep 19:46. https://doi.org/10.1007/s11934018-0796-X

7. Brunocilla $E$, Pultrone $C V$, Schiavina R, Rocca $C$ PassarettiG,CortiB etal (2012)Testicularsclerosing sertoli cell tumor: an additional case and review of the literature. Anticancer Res 32:5127-5130

8. Nuttall FQ, Warrier RS, Gannon MC (2015) Gynecomastia and drugs: a critical evaluation of the literature. Eur J Clin Pharmacol 71:569-578. https://doi.org/10.1007/s00228-015-1835-x

9. Deepinder F, Braunstein GD (2012) Drug-induced gynecomastia: an evidence-based review. Expert Opin Drug Saf 11:779-795. https://doi.org/10. 1517/14740338.2012.712109

10. Autorino $R$, Perdonà $S, D^{\prime}$ Armiento $M$, De Sio $M$, Damiano R, Cosentino Let al (2006) Gynecomastia in patients with prostate cancer: update on treatment options. Prostate Cancer Prostatic Dis 9:109-114. https://doi.org/10.1038/sj.pcan. 4500859

11. Bautista-Vidal C, Barnoiu O, García-Galisteo E, Gómez-Lechuga P, Baena-González V (2014) Treatment of gynecomastia in patients with prostate cancer and androgen deprivation. Actas Urol Esp 38:34-40. https://doi.org/10.1016/j. acuro.2013.02.013

12. Nguyen PL, Alibhai SMH, Basaria S, D'Amico AV, Kantoff PW, Keating NL et al (2015) Adverse effects of androgen deprivation therapy and strategies to mitigate them. Eur Urol 67:825-836. https://doi. org/10.1016/j.eururo.2014.07.010

13. Bedognetti D, Rubagotti $A$, Conti G, Francesca F, De Cobelli O, Canclini L et al (2010) An open, randomised, multicentre, phase 3 trial comparing the efficacy of two tamoxifen schedules in preventing gynaecomastia induced by bicalutamide monotherapy in prostate cancer patients. Eur Urol 57:238-245. https://doi.org/10.1016/j. eururo.2009.05.019

14. Fradet $Y$, Egerdie $B$, Andersen $M$, Tammela TLJ, Nachabe M, Armstrong J et al (2007) Tamoxifen as prophylaxis for prevention of gynaecomastia and breast pain associated with bicalutamide $150 \mathrm{mg}$ monotherapy in patients with prostate cancer: a randomised, placebo-controlled, dose-response study. Eur Urol 52:106-114. https://doi.org/10. 1016/j.eururo.2007.01.031

15. Viani GA, Bernardes da Silva LG, Stefano EJ (2012) Prevention of gynecomastia and breast pain caused by androgen deprivation therapy in prostate cancer: tamoxifen or radiotherapy? Int Radiat Oncol Biol Phys 83:e519-524. https://doi. org/10.1016/j.jjrobp.2012.01.036

16. Ghadjar P, Aebersold DM, Albrecht C, Böhmer D, Flentje M, Ganswindt U et al (2020) Treatment strategies to prevent and reduce gynecomastia and/or breast pain caused by antiandrogen therapy for prostate cancer. Strahlenther Onkol 196:589-597. https://doi.org/10.1007/s00066020-01598-9

17. Tunio MA, Al-Asiri M, Al-Amro A, Bayoumi $\mathrm{Y}$, Fareed M (2012) Optimal prophylactic and definitive therapy for bicalutamide-induced gynecomastia: results of a meta-analysis. Curr Oncol 19:e280-288. https://doi.org/10.3747/co. 19.993

18. McKeown SR, Hatfield P, Prestwich RJ, Shaffer RE Taylor RE (2015) Radiotherapy for benign disease; assessing the risk of radiation-induced cancer following exposure to intermediate dose radiation. Br J Radiol 88:20150405. https://doi.org/10.1259/ bjr.20150405

19. Mannu GS, Sudul M, Bettencourt-Silva JH, Tsoti SM, Cunnick G, Ahmed SF (2018) Role of tamoxifen 


\section{Buchbesprechung}

in idiopathic gynecomastia: a 10-year prospective cohort study. Breast J 24:1043-1045. https://doi. org/10.1111/tbj.13080

20. Boccardo F, Rubagotti A, Battaglia M, Di Tonno P, Selvaggi FP, Conti G et al (2005) Evaluation of tamoxifen and anastrozole in the prevention of gynecomastia and breast pain induced by bicalutamide monotherapy of prostate cancer. J Clin Oncol 23:808-815. https://doi.org/10.1200/ JCO.2005.12.013

21. Lefevre H, Bouvattier C, Lahlou N, Adamsbaum C, Bougnères $P$, Carel J-C (2006) Prepubertal gynecomastia in Peutz-Jeghers syndrome: incomplete penetrance in a familial case and management with an aromatase inhibitor. Eur J Endocrinol 154:221-227. https://doi.org/10.1530/eje.1. 02085

22. Rahmani S, Turton P, Shaaban A, Dall B (2011) Overview of gynecomastia in the modern era and the Leeds gynaecomastia investigation algorithm. Breast J 17:246-255. https://doi.org/10.1111/j. 1524-4741.2011.01080.x

23. Baumann K (2018) Gynecomastia-conservative and surgical management. Breast Care 13:419-424.https://doi.org/10.1159/000494276

24. Holzmer SW, Lewis PG, Landau MJ, Hill ME (2020) Surgical management of gynecomastia: a comprehensive review of the literature. Plast Reconstr Surg Glob Open 8:e3161. https://doi.org/ 10.1097/GOX.0000000000003161

25. Tripathy S, Likhyani A, Sharma R, Sharma RK (2020) Prospective analysis and comparison of periareolar excision (delivery) technique and pull-through technique for the treatment of gynecomastia. Aesthetic Plast Surg 44:653-661. https://doi.org/ 10.1007/s00266-020-01618-0

Note de l'éditeur. Springer Nature conserve une position neutre en ce qui concerne les revendications juridictionnelles dans les cartes géographiques et les affiliations institutionnelles figurant dans ses publications.

Bernhard Strauss, Marta Bertolaso, Ingemar Ernberg und Mina J. Bissell

\section{Rethinking Cancer}

A New Paradigm for the Postgenomics Era

Cambridge, United States of America: The MIT Press 2021, 432 S., (ISBN: 978-0262045216), 55,50 EUR

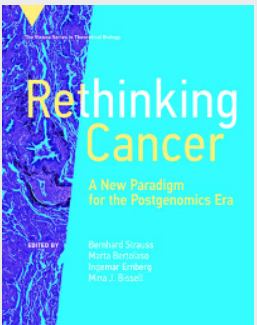

Führende Wissenschaftler erstellen einen neuen theoretischen Rahmen für die komplexe Erkrankung Krebs eine Grundlage zur Erforschung neuer Therapiekonzepte

Krebs ist eine äußerst komplexe Erkrankung, die weit über die genetischen Mutationen in der einzelnen Krebszelle hinausgeht. Trotz enormer Fortschritte im Verständnis der Krebsentstehung und der Etablierung neuer Therapieformen, wie der Immuntherapie, bleibt die Krebserkrankung eine klinische Herausforderung mit oft tödlichem Ausgang. Basierend auf empirischen Erkenntnissen präsentieren führende Wissenschaftler in diesem Buch einen neuen, theoretischen Rahmen, der als Grundlage für ein neues Kausalverständnis der Karzinogenese und zur Erforschung neuer Therapiekonzepte anwendbar ist.

Aus in sich geschlossenen konzeptionellen Bausteinen fügen die Autoren diesen Rahmen für eine umfassende neue Sichtweise auf die Erkrankung zusammen. Die Bausteine beschreiben zunächst theoretische Grundlagen, die zeitliche Dimension der Krebsprogression und die Notwendigkeit eines systembiologischen Ansatzes. Dabei werden Themen wie plastische molekulare Netzwerke, nichtkodierende DNA, Krebs als atavistischer Prozess, nichtlinearer Krankheitsverlauf, sowie Therapieabfolge und resistenz behandelt.
Ein weiterer Schwerpunkt liegt auf der Rolle der Mikro- und Makroumgebung, die entscheidenden Einfluss auf die Tumorzelle und damit den gesamten Krankheitsverlauf bis zu Metastasierung und Therapieresistenz hat. Dabei werden Themen wie der Einfluss von Gewebenischen, Metabolismus, Umweltfaktoren und Ernährung integriert und analysiert. Abschließend werden konkrete Schritte zur Umsetzung der konzeptuellen Bausteine in ein neues Paradigma für die Krebsforschung vorgeschlagen.

Das Buch ist ein Fundus für Anregungen zu neuen Denkmodellen in der Krebsforschung und spricht damit gleichermaßen grundlagenorientierte, translationale und klinische Krebsforscher an

Thomas Brabletz (Erlangen) 